\title{
Improving Construction Process through Integration and Concurrent Engineering
}

Malik M. A. Khalfan (Salford Centre for Research and Innovation (SCRI) in the Built and Human Environment, University of Salford, UK) and Naveed Raja (Salford Centre for Research and Innovation (SCRI) in the Built and Human Environment, University of Salford, UK)

\begin{abstract}
In an increasingly competitive business environment, improved time-to-market, reduced production cost, quality of the product and customer involvement are rapidly becoming the key success factors for any product development process. Consequently, most organisations are moving towards the adoption of latest technology and new management concepts and philosophies such as total quality management and concurrent engineering $(C E)$ to bring improvement in their product development process. This paper discusses the adoption of integrated processes and CE within the construction industry to enable construction organisations to improve their project development process. It also discusses a proposed integrated database model for the construction projects, which should enable the construction process to improve, become more effective and more efficient.
\end{abstract}

Keywords: concurrent engineering in construction, integration, integrated database model

\section{INTRODUCTION}

New advancements in information technologies and systems have created a very competitive business environment for all organisations across all sectors. In order to cope with this situation, construction organisations are moving towards the adoption of these new advancements in the technology to support their business environment accompanied by the implementation of new management concepts and practices such as concurrent engineering (CE). CE has the potential to make construction projects less fragmented, improve project quality, reduce project duration and reduce total project cost (Evbuomwan and Anumba, 1998; Kamara et al., 2000). This paper discusses the implementation of CE within the construction industry, and the integration of systems and teams to support CE implementation. Before discussing CE and its application to the construction industry, the integration of systems and teams will be discussed in the next section.

\section{INTEGRATION}

The continuous inter-disciplinary sharing of data, knowledge and goals among construction project participants is an integral process. It increasingly involves the linking together of two or more Information Technology (IT) systems to facilitate data transfer between them without the need for any re-input. The linkage of an IT system could be either intra-disciplinary (within one organisation) or inter-disciplinary (between two or more organisations). The purpose of integration should be aligned with an organisation's strategic objectives, which could be to increase business profit, improve market share, increase the size of their business market or create new markets. The other advantages of integration are: all staff having access to the latest data and information; more efficient storage of data; more easily maintained systems; rapid flow of information; improved decision making; improved co-ordination; better technical solutions due to effective feedback; and enhanced quality control and management due to improved procedures and monitoring (Peppard, 1993; Segars and Grovers, 1996).

\section{CONCURRENT ENGINEERING}

$\mathrm{CE}$, sometimes referred to as simultaneous engineering or parallel engineering, has been defined in several ways by different authors. The most popular one is that by Winner et al. (1988), who stated that CE "... is a systematic approach to integrated, concurrent design of products and their related processes, including manufacture and support. This approach is intended to cause the developers, from the outset, to consider all elements of the product life cycle from conception through disposal, including quality, cost, schedule, and user requirements".

\section{Concurrent Engineering in Construction}

In the context of the construction industry, Evbuomwan and Anumba (1998) defined CE as an "...attempt to optimise the design of the project and its construction process to achieve reduced lead times, and improved quality and cost by the integration of design, fabrication, construction and erection activities and by maximising concurrency and collaboration in working practices". This is in sharp contrast with the traditional approach to construction project delivery.

\section{Traditional Approach}

The traditional approach to many construction projects has been for the architect to produce an architectural design based on the client's brief. This architectural design is given to the structural engineer, who on completing the structural design passes the details to the quantity surveyor to produce the costing and bill of quantities. This goes on until the project is then passed on to the contractor who takes responsibility for the construction of the facility. This scenario, which is similar to the 'over the wall' approach (Evbuomwan and Anumba, 1998; Prasad, 1997), is shown in Figure 1.

Key disadvantages prevalent with this approach include:

- the fragmentation of the different participants in the construction project;

- the fragmentation of design and construction data;

- the occurrence of costly design changes and unnecessary liability claims;

- the lack of true life-cycle analysis of the project; and

- lack of communication of design rationale and intent. 


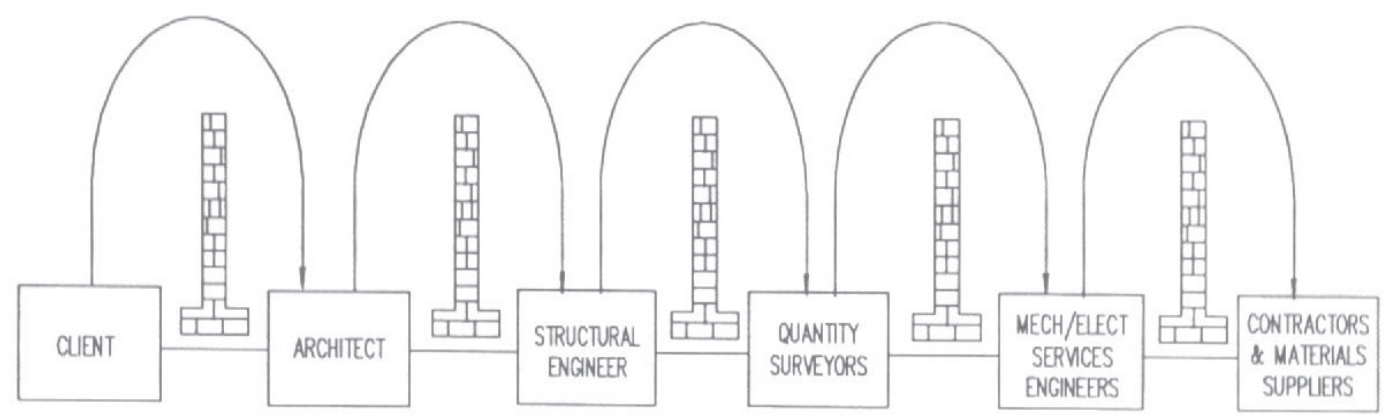

Figure 1: The over the wall approach (Evbuomwan and Anumba, 1998)

To address these issues, there is urgent need for a shift in paradigm within the construction industry. This should involve the adoption of new business strategies, with the aim of integrating the functional disciplines (see Figure 2) at the early stages of the construction project (Evbuomwan and Anumba, 1998).

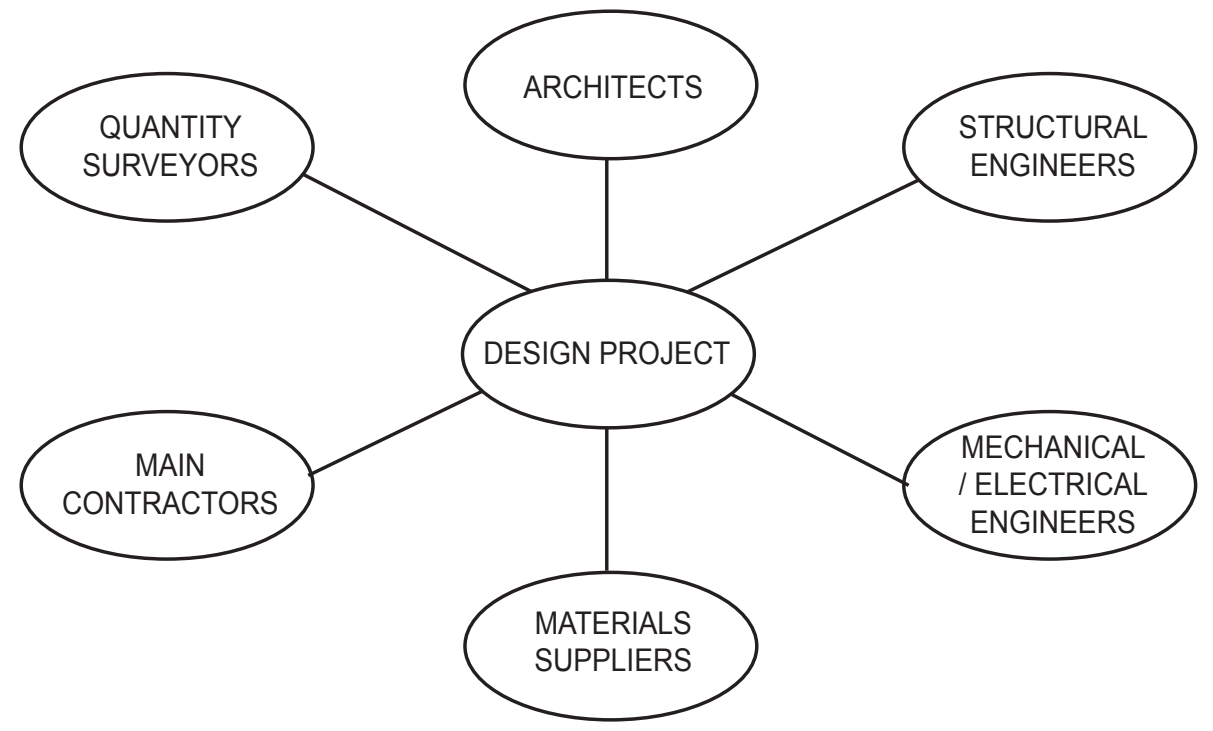

Figure 2: A typical project team (Evbuomwan and Anumba, 1998)

\section{Application of CE to Construction}

There is an urgent need to improve the performance of the construction supply chain participants (Evbuomwan and Anumba, 1998). This could be achieved during the design process by considering all aspects of the project's downstream phases concurrently. Incorporating requirements from the construction operation and maintenance phases at an early stage of a project would undoubtedly lead to an overall improvement in project performance. The essential constituents of 'concurrent construction' are as follows (Love and Gunasekaran, 1997; Chen, 1996):

- the identification of associated downstream aspects of design and construction processes;

- the need to bring in downstream specialists and subcontractors as early as possible in the design phase;

- the reduction or elimination of non-value adding activities; and

- the multi-disciplinary team.

\section{Why Adopt CE?}

There might be disagreement on the wording of definitions, or on whether CE is a philosophy, a methodology, an innovation or a strategy, but there tends to be a good consensus that CE is trying to: improve a company's business performance in the global marketplace by reducing the time-to-market; designing 'right first time' in order to reduce redesign of the product; reducing the costs of developing new products; and improving both quality and product variety. Other benefits of CE introduction and implementation include: improvement in communication, quality and production costs; and a reduction in design change and design repetition (Melling et. al., 1996).

\section{Integration - A Fundamental Aspect of CE}

CE includes a wide range of concepts, issues and aspects, with integration perhaps being the most important. In order to enable the CE practices to be implemented, the integration of IT systems, the integration of product development teams and their members and the integration of product data are all essential. In the following section, integration within the construction industry is discussed in detail. 


\section{INTEGRATION WITHIN THE CONSTRUCTION INDUSTRY}

The fragmented structure of teams within traditional construction business, as discussed in the previous section, does not support the implementation of CE practices. In order that the adoption of CE within the construction industry is effective, efficient and worthwhile requires the integration of construction teams in such a way that the team participants can share construction project data with each other.

\section{Team structure}

The project development team is usually responsible for supervising the whole project development process from the inception until hand-over. The members of a project development team often change over the life of a project and can be drawn from several construction organisations. As most of these organisations will have more than one ongoing project, a matrix management approach is required. The project development team oversees the performance of all other teams working on that project and should comprise representatives from all other related teams. Representatives could be either the team leader or any other person from that team such as the chief architect (Evbuomwan and Anumba, 1998). A typical team structure for a construction project is shown in Figure 3.

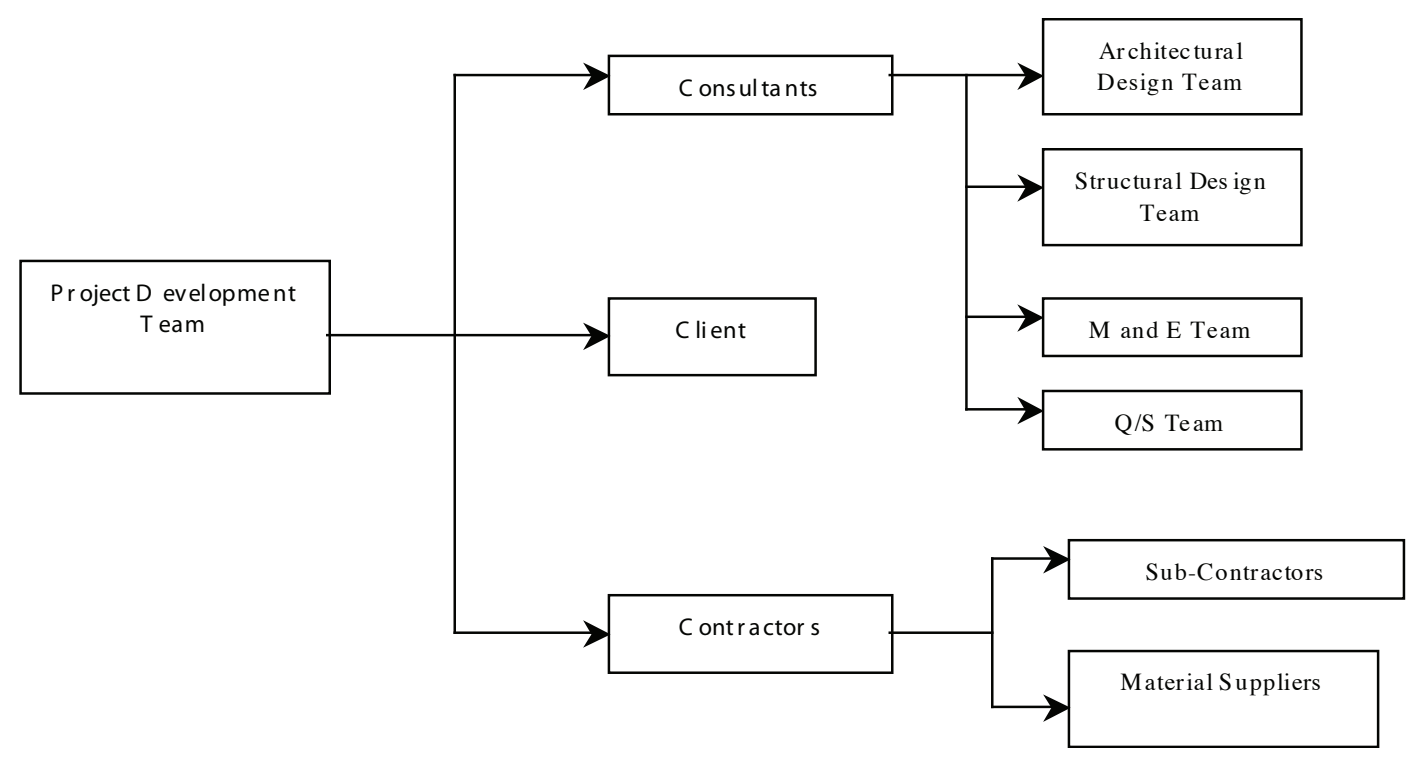

Figure 3: Typical team structure within a vonstruction organisation

(Khalfan and Anumba, 2000)

Most teams involved in the project development process are part of several organisations and not a single organisation. Teams of quantity surveyors, architectural designers and structural designers take part in a particular construction project through their organisation, which is specialist in that area. This type of arrangement could be considered as a virtual construction organisation if participants of the project development team and sub-teams operate from different locations. In this case, integration of these teams is vital in order to work together on one project. Integration of teams and systems is important so that they can share information and data with each other. An "integrated database model for the construction industry", which could provide a framework for the above mentioned integration requirements, has been developed by the authors and is described in the next section.

Integrated Database Model for the Construction Industry In this section, the model presented in Figure 4 is briefly described. The model illustrates: the integration across all the disciplines working for a particular project; integration in terms of sharing data; and communication with each other through an 'integrated database'. The integrated database (or shared construction project model) is a centralised database, which is accessible to all members of the project development team through their computers. All the participants can access the information and data related to the project and can also send their own progress update to this database. This is a move towards inter-disciplinary collaboration. The centralised database would be accessed in order to monitor the progress and current status of the project. This database should be available from the outset of the project to enable all members of the project development team to view and contribute towards design simultaneously. This would enable inputs from all project participants to be taken into account and design details made 'right first time'. This will help to avoid redesign of the later stages of the project and supports the CE practice of working simultaneously within a team towards one goal. Similarly, there could be intra-disciplinary collaboration. This has been shown in Figure 4, where the structural design team has several sub-teams all contributing the structural design. A centralised integration of their individual designs is being performed within the structural design team. The integrated design, which results from the work done by structural design team and architectural design team, is then sent to the centralised or integrated database for the other project participants. This intra-disciplinary collaboration can also be seen in other teams (Amor and Anumba, 1999; Anumba, 1996; Anumba and Duke, 1997; Evbuomwan and Anumba, 1996; Henry, 1993; Laudon and Laudon, 1998; Laudon and Laudon, 1996; Sudweeks and Allbritton, 1996). 


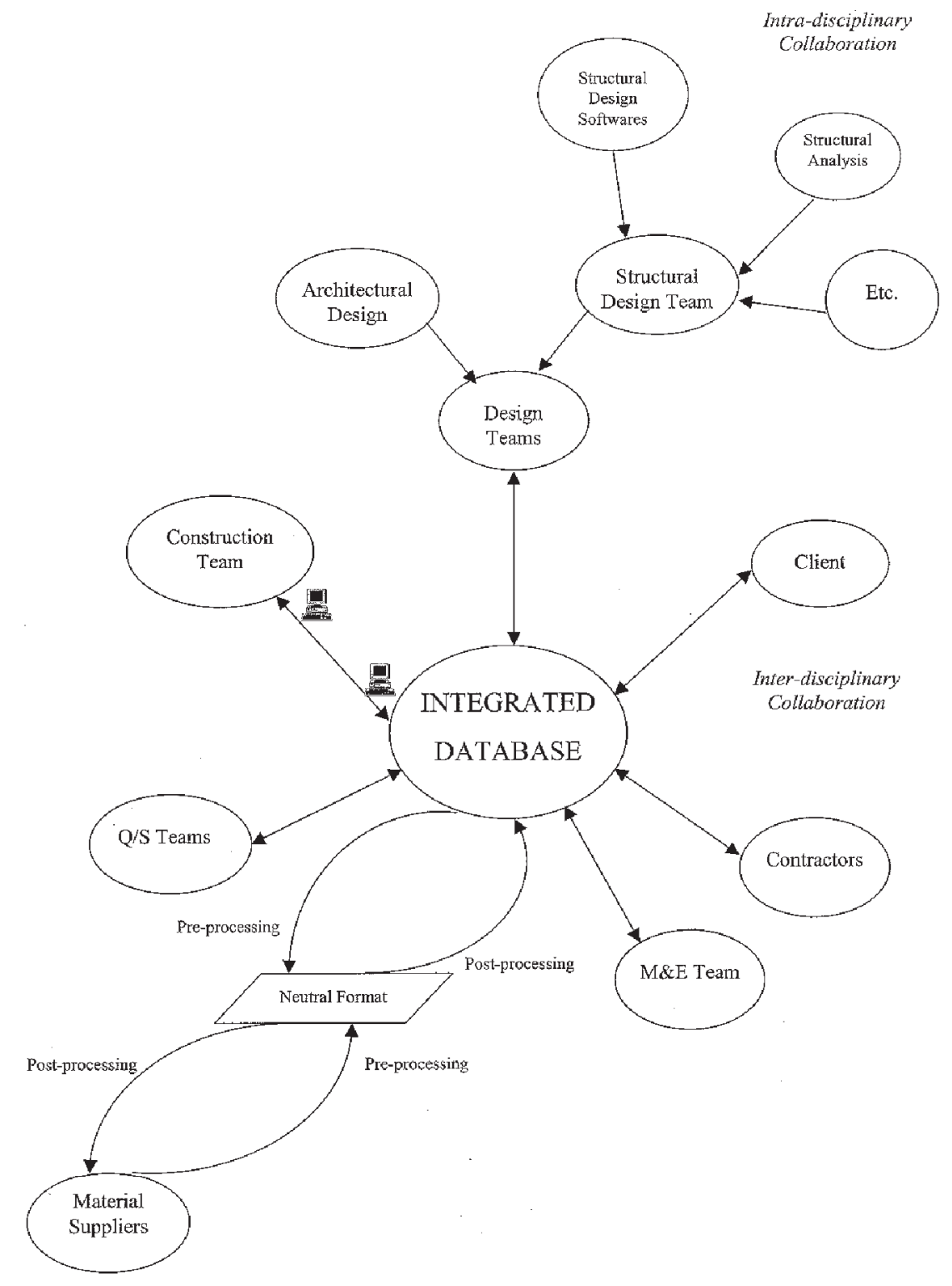

Figure 4: Integrated database model for the construction industry

The model supports the 'neutral file format' of all data and information. If the material suppliers have recorded their data in file format $A$, but the data in the integrated database is in file format $B$, there is a facility within this model which supports neutral format of the data, which could be performed as shown in the model. Preand post-processing operations are available which can convert file format $A$ into $B$ and vice versa, supporting the integration of different operating and filing systems (Laudon and Laudon, 1998). The adoption of the Industry Foundation Classes (IFCs), being developed by the International Alliance for Interoperability (IAI) (see their website for further details: http://www.iai-international. org/iai_international/), will facilitate future interpretability between systems, making the use of the neutral file format unnecessary.

As discussed earlier, the project development team participants can be located within different organisations. There is a possibility that the project construction site is located in Birmingham and the architectural team is based in Manchester or the structural designer team has their office in Loughborough. In order to bring them closer and support integration during the construction stage of the project, multi-media technology could be used both on site and in other participants' offices. Video conferencing and other multi-media technologies are being used to support the integration of the entire project information and data across all the participants of project development team during the construction phase of a project (Miah et al., 1998; Thorpe et al., 1995).

Recent developments in the fields of information technology and business information systems, such as virtual reality and intelligent agents, could be included in the proposed model to enable project development processes to be more effective and efficient. Advancements in the field of virtual reality have been adopted within construction project development. The architectural designers, on the basis of the detailed design, could develop a virtual model of the project. This model could be kept at a centralised location within an integrated database and the progress of the project could be represented with this virtual model. The virtual model would also help other participants of the project development team to identify any specific problems related to constructability, feasibility, durability and availability of material. It also helps the client specifically to judge that this virtual model is according to the projects requirements and needs (Roger, 1994).

Similarly, intelligent agents could be installed in all the computers within all the teams and at the centralised location in the model. The intelligent agent would inform all the team participants if there 
were any changes to design details, so that all the participants take these changes into account and up-date their work. For example, if there were any changes to the architectural design, the intelligent agent of the architectural design team would inform all other intelligent agents of other teams and also update the centralised database. All the team members would be informed by their respective agents and changes could be viewed via the integrated database and hence the team members could make changes to their work (Anumba and Newnham, 1998; Melham, 1999).

\section{Examples from the Construction Industry}

The examples of the applications of the above concept within industry are taken from a TCS (Teaching Company Scheme) project funded by the DTI. The partnership was between Property Tectonics and the University of Salford. Property Tectonics is a small construction consultancy with two offices, one in Manchester and the other in St Albans. The issues of intra-disciplinary integration and sharing of knowledge and data are shown in Case Study 1 and issues of inter-disciplinary integration are discussed in Case Study 2.

\section{Case Study 1 (Intra-disciplinary)}

Implementation of a VPN (Virtual Private Network)

The two offices of Property Tectonics are located at different regions of the country, the main office being in Manchester and the other in St Albans. The offices previously used to connect to each other using a modem to send e-mails and other documents. This was a time-consuming process as some documents were large CAD drawings. Another major problem was that the SAGE (Accounting, payroll and job costing software) database was stored on the main server in the Manchester office and the other office could not access or use this information.

A proposal was been made to implement a permanent communications connection via a VPN (Virtual Private Network) between the two offices (see Figure 5). A VPN is a private network constructed using the public network, the Internet, as the medium for transporting data. This option was chosen over using Leased Lines (a permanent telephone connection between 2 points) because it was significantly cheaper. The installation of ADSL broadband with 2 firewalls, one at each office was carried out. The VPN and the servers were configured to communicate with each other. The "always on" ADSL broadband connection meant it was acting like a permanent leased line connection but without the associated costs.

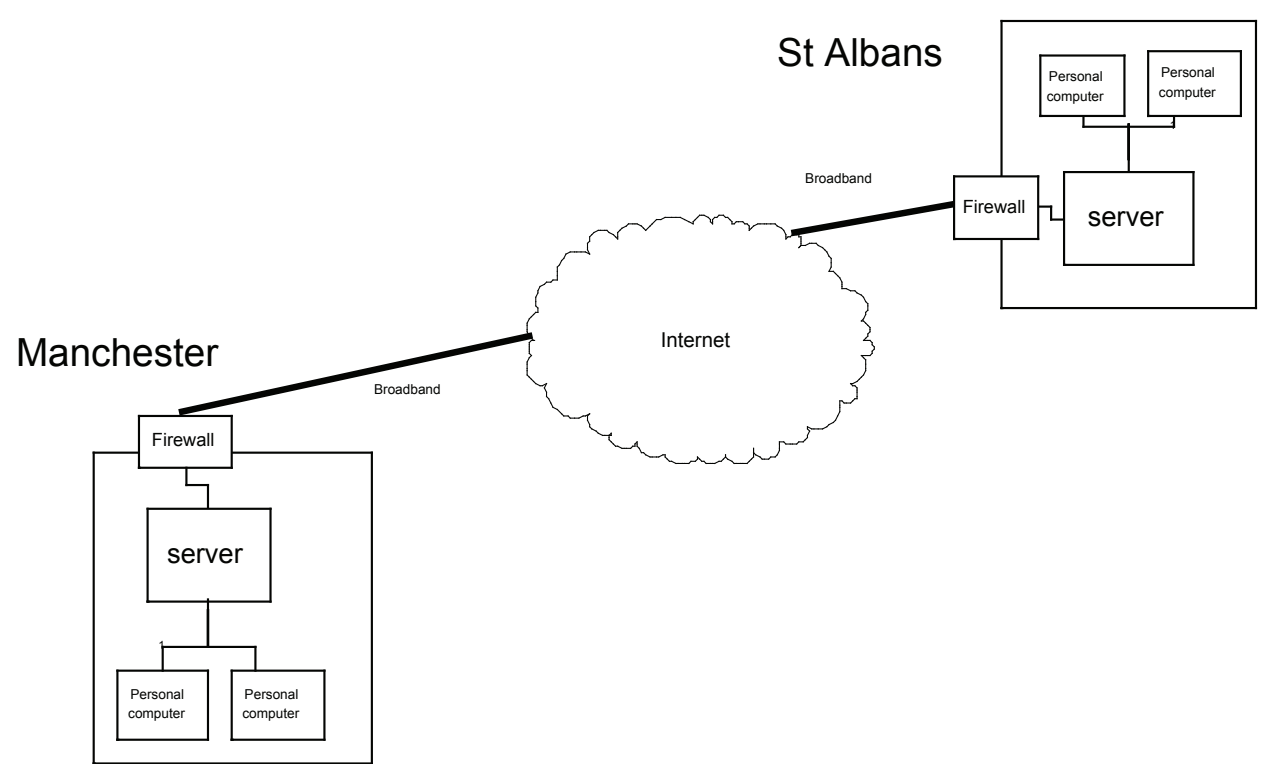

Figure 5: VPN (Virtual Private Network) between two offices

The use of the VPN enabled each office to 'see' what was stored on the server in the other office. There was no longer any need to e-mail documents as a user from one office can open the file as if it is stored locally. Other advantages include sharing software and being able to use, for example, the SAGE accounting software that is only stored in one office. Staff from the other office can have access to this and can therefore now do their own accounts. This type of network means that although the companies are geographically separated they work as if they are all based at one site. They have access to all the documents and software tools. This enables the workers to be more productive because they do not have to wait for a colleague to send documents or update the accounts from the other office. They now have access to these facilities themselves. The connection is also secure because there are firewalls in place to only allow access to known people and IP addresses (an identifier for a computer). The above case study shows an example of the use of an integrated system, which is enabling people from the same organisation to work concurrently and in a collaborative manner while working on the same project from different locations.

\section{Case Study 2 (Inter-disciplinary)}

The PracticeNet Model

The Practice Network (PracticeNet) is a cross organisational integration initiative. The vision is to create an integrated, dynamic, solid but flexible system to provide a quality, valueadded service to the customer, by way of a national network of consultancies. The network consists of small and medium size surveying consultancies that will provide coverage throughout the country. There are six companies that make up the PraticeNet strategic partnership. They are based in Manchester, Northern Ireland, Coventry, Edinburgh, Cardiff and London. The alliance will take the partnering companies into new markets and give them the ability to engage in the size of contracts that have previously been dominated by only the largest of companies. 
The aim is to facilitate collaboration between groups of people who are working together but are geographically spread. This would enable better communication between offices and colleagues. The members of the PracticeNet system have remote access to information. This idea is to have everything stored centrally, on a web-enabled centralised database. The ERD (Entity Relationship Diagram) model was adopted to allow the creation of database tables that work well together in the system. This is a graphical representation of entities and their relationships to each other, to organise the data within a database system. An entity is an object about which data is stored and the relationship would show how this data is shared between the entities.

The quality assurance (QA) forms that are being used, as online forms, were first considered. The data from these online forms needed to be stored and manipulated for data interrogation. The design of the database is such that it can take the information from the forms, and link them to information from the remaining QA forms which are all related through a job number. Every project taken on is allocated an individual job number.
The forms have been broken down into the components parts, which make up the database design. Each of the forms have been allocated different tables and then these have been linked via the relationships. These relationships have been identified using the ERD model. Information stored on a central server means there is no need for anyone to download documents or to have compatible software to be able to view the documents. The only requirement is to have an Internet connection, which most companies already have. Access to the information stored on the central server is via username and password. Initially all information was available as view only for users with access to the system. This is to build more trust between the partnering companies.

The home page and other screens presented to the users is user friendly, built with good graphical user interface techniques in mind. The screens consist of prompts and clickable items to open other pages and links for more information (see Figure 6). This shows a marketing request form; once information on a job has been entered the buttons on the left would show the information relating to the different stages of the project and its progress.

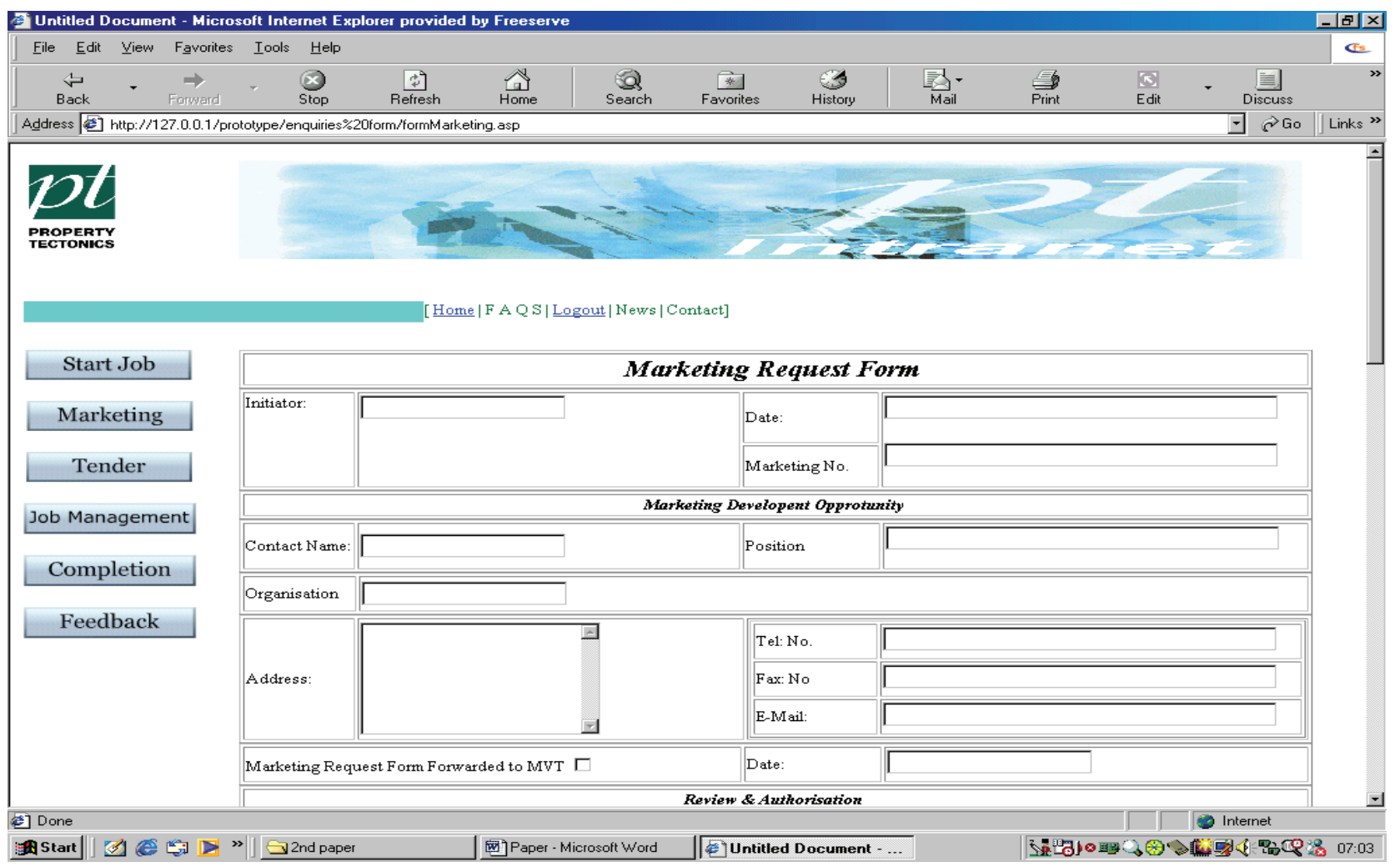

Figure 6: Marketing request form

A process map has been developed (see Figure 7). This was done using the research carried out by the Process Protocol Team at the University of Salford. The process protocol map defines the different stages and people involved in a project lifecycle. A link to the different stages of this map will bring up a graphical representation of that stage. From here the user can select the required process that is involved within each of the stages by clicking on the relevant section of the map. This has a hyperlink to the relevant database, instructions, or documentation. The user can then complete or browse the information that they need. If a requirement is for a form to be completed then once this is done the submit button sends the results and stores them in the database held at the central server. 


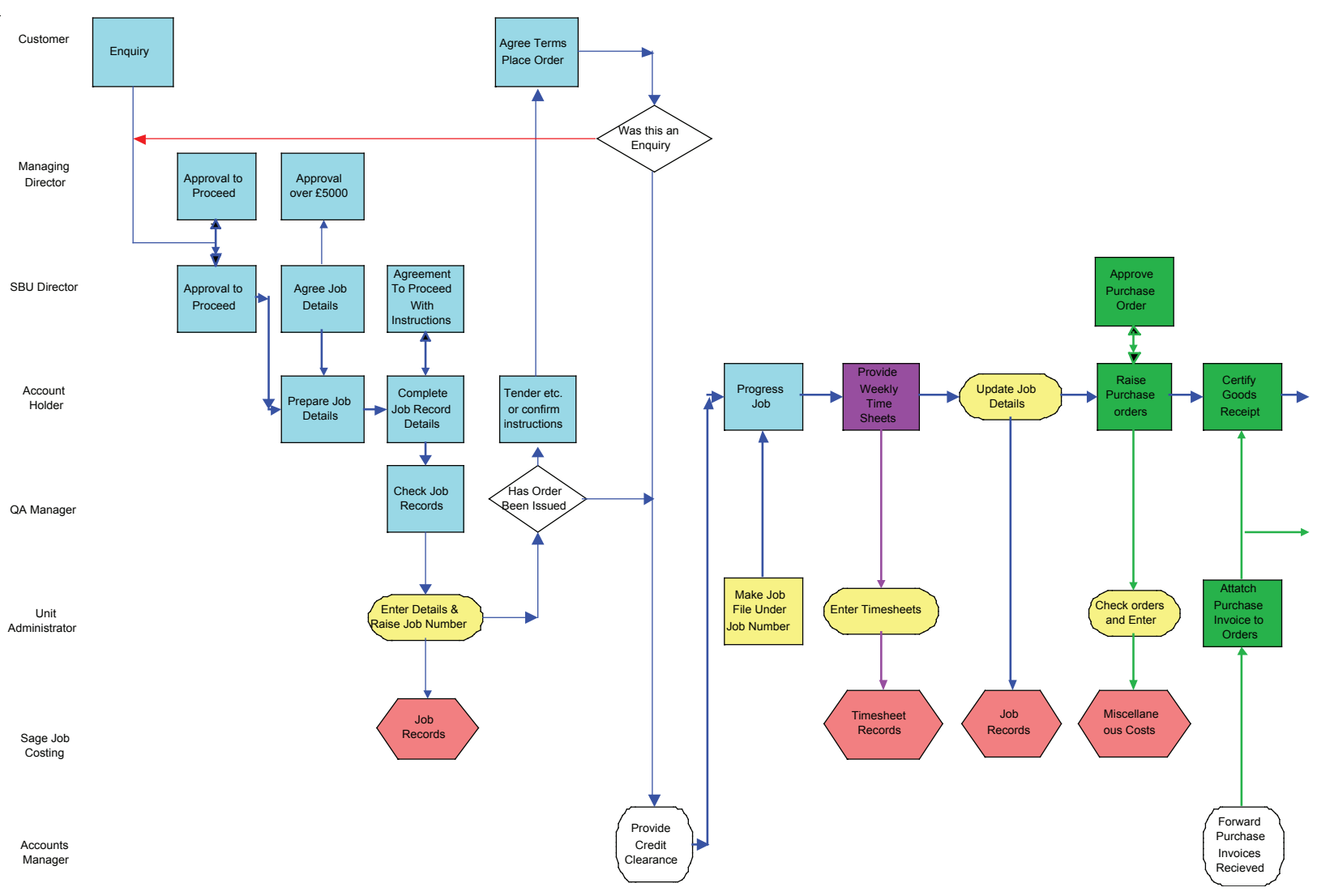

Figure 7: A diagram of the process map [part (a)]

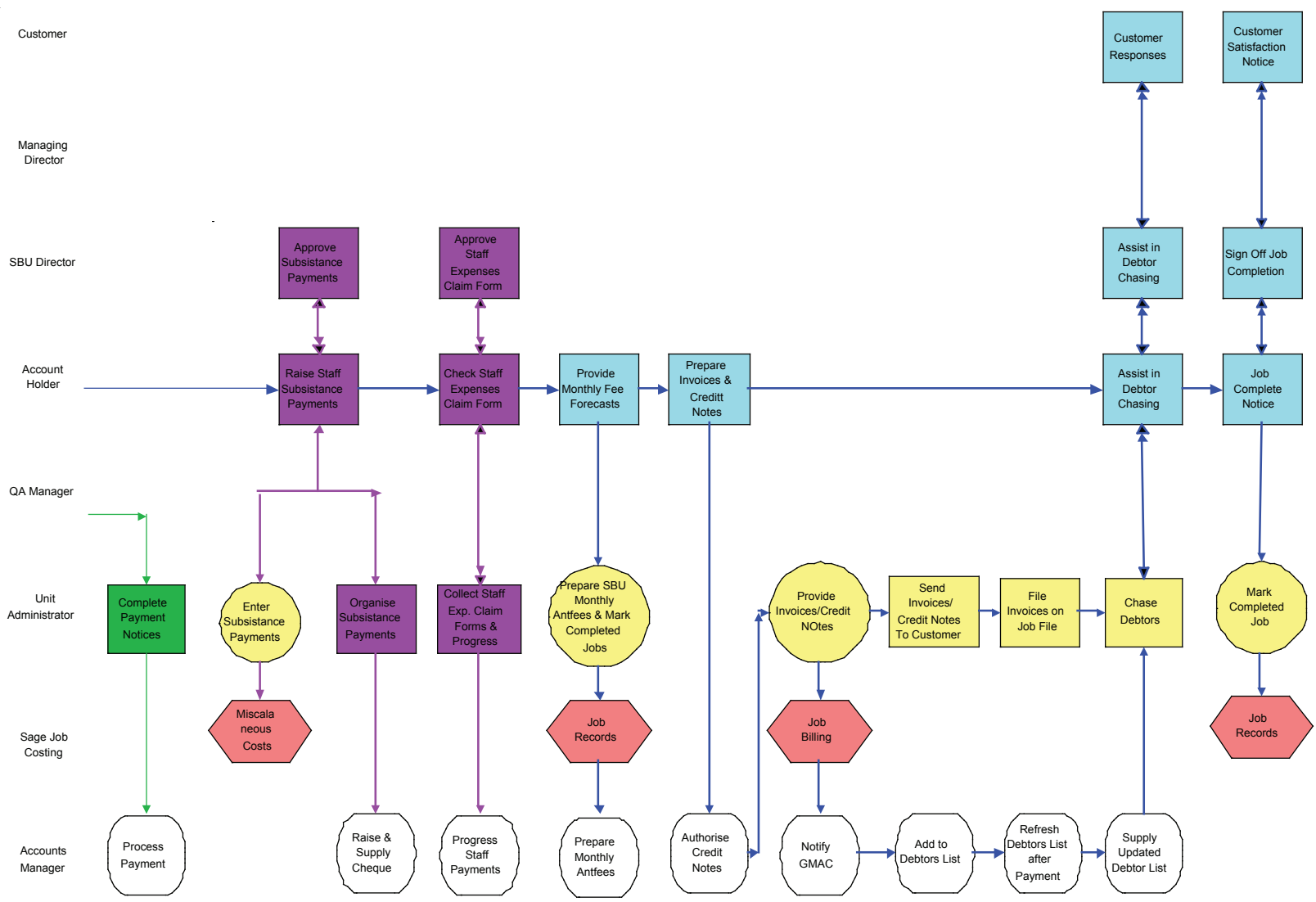

Figure 7: A diagram of the process map [part (b)] 
A job number is used to categorise jobs. From this the user can retrieve information about the progress of the job related to this job number. Any information or additional details that need to be added are saved according to this job number.

Collaborative software provides the ability for members of a team, such as planners, designers or architects to collaborate and share documents, allocate tasks, share a calendar that shows when they are busy, etc. This information is used instead of e-mailing documents for the arrangement of meetings. Each member of the team have access to the current version of any ideas, sketches, documents and other material that have been posted online for group access. This means that if someone has made any changes to the document then all members are aware of this, and will always work on the current version.

Other useful information is also available such as a view of upto-date ISO 9001 work instructions, maintenance procedures, and company policy, quality manuals, newsletters and announcements or the latest press release.

All the above shows that there are benefits of using integrated IT systems by different companies, which are involved in a same project but are located at different places geographically. This also enables the partnering companies to work as an integrated team and work on the project simultaneously, and in a collaborative manner.

PracticeNet is a new and innovative entrant to the UK property and construction markets. With a total of 300 professional staff working from 25 offices, PracticeNet offers complete geographical coverage of both the UK and Ireland. With over 109 building surveyors nationally, PracticeNet is now one of the top suppliers of building surveying services within the UK. PracticeNet will be looking for a European partner as part of an EEIG (European Economic Interest Group) to expand and provide coverage of services within the European market.

\section{SUMMARY AND CONCLUSIONS}

An integrated and concurrent project environment could help construction organisations to grow and become more competitive in a highly competitive business climate. The use of new advancements in business information technology such as virtual reality, intelligent agents, multi-media and video conferencing is critical to achieving an integrated and concurrent project environment. The appropriate use of these business information systems within the construction industry will bring about a virtual construction project improving co-ordination, visualisation and construction supply chain management. This in turn should result in increased profitability, higher quality of projects, reduced construction time and cost, increased client involvement, better project performance and improved client satisfaction. This should help the construction industry to move towards the targeted objectives as set by Egan $(1998,2002)$ and Latham (1994).

\section{REFERENCES}

Amor, R. and Anumba, C.J. (1999), 'A Survey and Analysis of Integrated Project Databases', in proceedings of Concurrent Engineering in Construction, Bath, UK, September 1999, pp.217227.

Anumba, C.J. (1996), 'Functional Integration in CAD Systems', Advances in Engineering Software, vol.25, pp.103-109.
Anumba, C.J., Duke A. (1997), 'Structural Engineering in Cyberspace: Enabling Information and Communication Technologies', The Structural Engineer, vol.75, no15, pp.259-263.

Anumba C.J, Newnham L. (1998), 'Towards the use of Distributed Artificial Intelligence in Collaborative Building Design', in proceedings of First International Conference on New Information Technologies for decision Making in Civil Engineering, Miresco, E. T. (ed.), Montreal, Canada, 11-13 Oct., pp.413-424.

Chen, G. (1996), 'The Organisational Management Framework for Implementation of Concurrent Engineering In the Chinese Context, Advances in Concurrent Engineering', in proceedings of 3rd ISPE International Conference on Concurrent Engineering: Research and Applications, University of Toronto, Ontario, Canada, 26-28 August 1996, pp.165-171.

Egan, J. (1998), 'Rethinking Construction', report of the Construction Task Force on the Scope for Improving the Quality and Efficiency of UK Construction Industry, Department of the Environment, Transport and the Regions, London.

Egan, J. (2002), 'Accelerating change', report by Strategic Forum for Construction, 2002, London.

Evbuomwan, N.F.O. and Anumba, C.J. (1998), 'An Integrated Framework for Concurrent life-cycle Design and Construction', Advances in Engineering Software, 1998, vol.5, no.7-9, pp.587597.

Evbuomwan, N.F.O. and Anumba, C.J. (1996), 'Towards an Integrated Engineering Design Environment', Information Representation and Delivery in Civil and Structural Engineering Design, Kumar, B. and Retik, A. (eds.), Civil-Comp Press, pp.127134.

Henry, R.M. (1993), 'Role of Advanced Computer Technology in Construction Industry', Journal of Computing in Civil Engineering, vol.8, no.3, July 1994.

Kamara, J.M.; Anumba, C.J. and Evbuomwan, N.F O. (2000), 'Developments in the Implementation of Concurrent Engineering in Construction', International Journal of Computer Integrated Design and Construction, vol.2, no.1, Feb. 2000, pp.68-78.

Khalfan, M.M.A. and Anumba, C.J. (2000), 'Implementation of Concurrent Engineering in Construction - Readiness Assessment', in Proceedings of Construction Information Technology (CIT2000) Conference, Reykjavik, Iceland, 28-30 June 2000, vol.1, pp.544-555.

Latham, M. (1994), 'Constructing the Team', Final Report on Joint Review of Procurement and Contractual Agreements in the UK Construction Industry, HMSO, London.

Laudon, K.C. and Laudon, J.P. (1998), Management Information Systems: New Approaches to Organisation and Technology, 5th Ed. Prentice Hall, New Jersey.

Laudon, K.C. and Laudon, J.P. (1996), Management Information Systems: Organisation and Technology, 4th Ed., Prentice Hall, New Jersey.

Love, P.E.D. and Gunasekaran, A. (1997), 'Concurrent Engineering in the Construction Industry', Concurrent Engineering: Research and Applications, vol.5, no.2, June 1997, pp.155-162. 
Melham A (1999), Intelligent Agents. Available at Construction-IT web site, (http://www.construct-it.salford.uk).

Melling, T., Lomax, J., Cather, H. and Koshal, D. (1996), 'The Use of Simulation Techniques in the Implementation of Concurrent Engineering in Small to Medium Sized Enterprises', Advances in Concurrent Engineering, in proceedings of 3rd ISPE International Conference on Concurrent Engineering: Research and Applications, University of Toronto, Ontario, Canada, 26-28 August 1996, pp.352-359.

Miah T., Carter C., Thorpe A., Baldwin A. and Ashby S. (1998), 'Wearable Computers - an application of BT's mobile video System for the construction industry', BT Technology Journal, vol.16, no.1, Jan 1998

Peppard, J. (1993), IT Strategy for Business, Pitman, London.

Prasad, B. (1997), 'Seven Enabling Principles of Concurrency and Simultaneity in Concurrent Engineering, Concurrent Engineering in Construction, in proceedings of 1st International Conference organised by The Institution of Structural Engineers Informal Study Group on Computing in Structural Engineering, 3 and 4 July 1997, London, pp.1-12.

Roger, A. S. (1994), 'Virtuosi - Virtual Reality Support for Groupworking', BT Technology Journal, vol.12, no.3, July 1994.

Segars, A. H. and Grovers, V. (1996), 'Designing Company-wide information system', Long Range Planning, vol.29, no.3, pp.381392.

Sudweeks, F. and Allbritton, M. (1996), 'Working Together Apart: Communication and Collaboration in a Network Group', in proceedings of 7th Australasian Conference of Information Systems, Hobart, Tasmania, December 11-13, vol.2, pp.701-712.

Thorpe A., Baldwin A., Cater C., Leevers D. and Madigan D. (1995), 'Multimedia Communications in Construction', in proceedings of the Institution of Civil Engineers, 1995, vol.108, no.1, pp.12-16.

Winner, R.I., Pennell, J.P., Bertrend, H.E. and Slusarczuk, M.M.G. (1988), 'The Role of Concurrent Engineering in Weapons System Acquisition', IDA Report R-338, Institute for Defence Analyses, Alexandria, VA, U.S.A. 